\title{
ON OPTIMAL PARAMETRIC CONTROL OF PARABOLIC SYSTEM
}

\author{
Jan Sokołowski \\ Institute for Organization, Management \\ and Control Sciences \\ 00-818 Warszawa, ul. KRN, Poland
}

\section{Introduction}

In the paper we consider a parametric optimization problem for an abstract parabolic equation. Problems of such a type are investigated in the case of elliptic equations in $[2],[10]$ and parabolic equations in $[2],[3],[5],[11]$. In this paper two types of observations : in spaces $\mathrm{C}(\mathrm{O}, \mathrm{T} ; \mathrm{H})$ and $\mathrm{C}(\mathrm{O}, \mathrm{T} ; \mathrm{V})$ are considered.

For both cases sufficient conditions of existence of an optimal control are given. Using the so called generalized adjoint state equation necessary conditions of optimality are formulated. Proofs of presented results are given in $[7],[8],[9]$. Let there be given Hilbert spaces V, H with $/ 1.1 / \mathrm{V}<\mathrm{H}, \mathrm{V}$ dense in $\mathrm{H}$ By Uad we denote the set of admissible controls which is assumed to be a convex subset of a Hilbert space $U$. Let there be given a family of bilinear forms on $V$ : $/ 1.2 / \quad a_{u}(t ; y, z)$ 
624

where

$$
\begin{aligned}
& u \leqslant U_{a d} \\
& t \in[0, T] \\
& y, z \in V
\end{aligned}
$$

We assume:

(i) family $/ 1,2 /$ is continuous on $V$, that is

$$
\begin{aligned}
& 11.3 / \quad\left|a_{u}(t ; y, z)\right| \leqslant M\|y\|_{V}\|z\|_{V}, \forall t \in[0, T], \\
& \forall u \in u_{a d}, \quad \forall y, z \in V
\end{aligned}
$$

(ii) napping

$$
\mathrm{t} \longrightarrow \mathrm{a}_{\mathrm{u}}(: ; \mathrm{y}, \mathrm{z})
$$

is measurable for all $y, z \in V$ and all $u \in U_{a d}$ with respect to Levesque measure on the interval $[0, \mathrm{~T}]$

(iii)

$/ 1.4 /$

$$
\begin{aligned}
& a_{u}(t ; y, y) \geqslant \alpha\|y\|_{V}^{2}, \quad \alpha>0, \\
& \forall u \in u_{a d}, \quad \forall t \in[0, T], \quad \forall y \in v
\end{aligned}
$$

Following $[3]$ we denote by $W(0, T)<L^{2}(0, T ; V)$ a Hilbert space with the scalar product:

$/ 1.5 /\left(y_{z},\right)_{W(0, T)} \int_{0}^{T}\left\{\left(\frac{d y}{d t}, \frac{d z}{d t}\right)_{v^{\prime}}+(y, z)_{V}\right\} d t$

where (., .) $V$ denotes scalar product in $V$.

Let there be given elements $y_{0} \in H$ and $f \in L^{2}\left(0, T ; V^{\prime}\right)$.

For given control $u \in U_{a d}$ we define state trajectory $y_{u} \in W(O, T)$

as the solution of an abstract parabolic equation of the form:

$$
\begin{aligned}
/ 1.6 / & \left(\frac{d y_{u}}{d t}(t), z\right)_{v v}+a_{u}\left(t ; y_{u}(t), z\right) \\
= & \left.(t(t), z)_{v v}, \quad \forall z \in v, \quad a . e, \text { in }\right] 0, T[
\end{aligned}
$$


/1.7/ $\quad \mathrm{y}_{\mathrm{u}}(0)=\mathrm{y}_{\mathrm{o}}$

where $(., \cdot)_{V}$ denotes scalar product between $\mathrm{V}^{\prime}$ and $\mathrm{V}$. Under the above assumptions problem $/ 1.6 /, / 1.7 /$ has the unique solution [4] which continuously depends on the data $y_{0} \in H$ and $P \in L^{2}(0, T ; V)$.

\section{Observation in $\mathrm{C}(0, \mathrm{~T} ; \mathrm{H})$}

Let us define on the set $U_{a d} \subset U$ the cost functional

$/ 2.1 /$

$$
J(u)=\frac{1}{2}\left\|y_{u}(T)-z_{d}\right\|_{H}^{2}+\frac{\varepsilon}{2}\|u\|_{U}^{2}
$$

where

$$
\mathrm{y}_{\mathrm{u}}(\mathrm{T})=\left.\mathrm{y}_{\mathrm{u}}\right|_{t=T}
$$

and $z_{d} \in H$ is a given element.

We shall consider the following minimization problem :

/2.2/ find $\inf _{u \in U_{a d}} J(u)$

\section{Lemma :}

If we assume that :

(i) bilinear form $/ 1.2 /$ is Lipschitzian with respect to u, that is

$$
\begin{aligned}
& \text { /2.3/ }\left|a_{u 1}(t ; y, z)-a_{u 2}(t ; y, z)\right| \leqslant c\left\|u_{1}-u_{2}\right\|_{U}\|y\|_{V}\|z\|_{v} \\
& \forall t \in[0, T], \quad \forall u_{1}, u_{2} \in U_{a d}, \quad \forall y, z \in v \\
& \text { (ii) } \quad \text { set } U_{a d} \in U \text { is compact }
\end{aligned}
$$

then there exists a solution $u \in U_{\text {ad }}$ to the problem /2.2\%

Proof is given in [8].

Let us assume that at the point $u \in U_{a d}$ there exists Frechet de- 
rivative $\tilde{A}_{u}(\hat{u})$ of the operator $\tilde{A}(u) \in \mathcal{L}\left(L^{2}(0, T ; V) ; L^{2}\left(0, T ; V^{\prime}\right)\right)$ which is defined by the equality

$$
\begin{aligned}
/ 2.4 / & ((\tilde{A}(\mathrm{u}))(\mathrm{t}) \mathrm{y}, \mathrm{z})_{V^{v}}=a_{u}(t ; y, z) \\
\forall u \in U_{a d}, & \forall t \in[0, T], \quad \forall y, z \in V
\end{aligned}
$$

To obtain a simple form of necessary conditions of optimality we introduce adjoint state $p_{u} \in W(0, T)$ which is defined as the soLution of adjoint state equation :

$$
\begin{gathered}
/ 2.5 / \quad-\left(\frac{d p_{u}}{d t}(t), z\right)_{V^{\prime} v}+a_{u}\left(t, z, p_{u}(t)\right)=0 \\
\forall z \in V, \quad a . e \text {. in }]_{0}, T[
\end{gathered}
$$

$12.6 /$

$$
p_{u}(T)=-y_{u}(T)+z_{d}
$$

optimal control $\hat{u} \in U_{\text {ad }}$ is characterized $[2],[8]$ by inequality :

$$
/ 2.7 / \int_{0}^{T}\left(\left\langle\tilde{A}_{u}(\hat{u}), u-u\right\rangle y_{\hat{u}}(t), p_{\hat{u}}(t)\right)_{v^{\prime} v} d t \geqslant 0,
$$

\section{Observation in $\mathrm{C}(\mathrm{O}, \mathrm{T} ; \mathrm{V})$}

In order to consider the case where cost dunctional is defined on $\mathrm{C}(\mathrm{O}, \mathrm{T} ; \mathrm{V})$ we have to use some representation of bilinear form $/ 1.2 /$. To do that we need some additional definictions.

Let there be given a Hilbert space $S$ and linear, bounded operator $\gamma \in \hat{\alpha}(V, s)$. We assume

/3.1/ operator $\gamma$ maps $v$ onto $s$

/3.2/ kernel ker $y=V_{0}$ is dense in $H$

It is easy to show that for given element $\varphi \in L^{2}\left(0, T ; S^{\prime}\right)$ linear runctional 
/3.3/ $\quad V \rightarrow z \longmapsto(\varphi(t), \gamma z)_{S^{\prime} S} \in R^{1}$

is continuous a.e. in $] 0, \mathrm{~T}[$.

With the form / $1.2 /$ we associate the so called formal operator :

/3.4/ $\quad A(u) \in \mathcal{L}\left(\mathrm{L}^{2}(0, \mathrm{~T} ; \mathrm{V}) ; \mathrm{L}^{2}\left(0, \mathrm{~T} ; \mathrm{V}_{0}^{\prime}\right)\right)$

which is defined by the formula

$$
\begin{gathered}
\text { /3.5/ } \int_{0}^{\mathrm{T}} \mathrm{a}_{\mathrm{u}}(\mathrm{t}, \mathrm{y}(\mathrm{t}), \mathrm{z}(\mathrm{t})) \mathrm{dt}=\int_{0}^{\mathrm{T}}((\mathrm{A}(\mathrm{u}))(\mathrm{t}) \mathrm{y}(\mathrm{t}), \mathrm{z}(\mathrm{t})) \mathrm{v}_{0}^{\prime} \mathrm{v}_{0} \mathrm{dt} \\
\forall \mathrm{z} \in \mathrm{L}^{2}\left(0, \mathrm{~T} ; \mathrm{V}_{0}\right), \quad \forall \mathrm{y} \in \mathrm{L}^{2}(0, \mathrm{~T} ; \mathrm{V})
\end{gathered}
$$

Furthermore we assume that the domain ${ }^{x /}$ of operator $A(u)$ considered as an unbounded operator in $L^{2}(O, T ; H)$ is a space $L^{2}(O, T ; D)$ where $\mathrm{DCV}$ is a given Hilbert space.

It can be shown [1] that there exists the unique operator, called Neumann operator :

/3.6/ $\sigma(u) \in \mathcal{L}\left(\mathrm{L}^{2}(0, \mathrm{~T} ; \mathrm{D}) ; \mathrm{L}^{2}\left(0, \mathrm{~T} ; \mathrm{s}^{\prime}\right)\right)$

such that the following representation takes place :

$$
\begin{aligned}
& / 3.7 / \quad \int_{0}^{\mathrm{T}} \mathrm{a}_{\mathrm{u}}(\mathrm{t} ; \mathrm{y}(\mathrm{t}), \mathrm{z}(\mathrm{t})) \mathrm{dt} \\
&=(\mathrm{A}(\mathrm{u}) \mathrm{y}, \mathrm{z})_{\mathrm{He}}+((\sigma(\mathrm{u}) \mathrm{y}, \tilde{\gamma} \mathrm{z})) \\
& \forall \mathrm{y} \in \mathrm{L}^{2}(\mathrm{O}, \mathrm{T} ; \mathrm{D}), \quad \forall \mathrm{z} \in \mathrm{L}^{2}(\mathrm{O}, \mathrm{T} ; \mathrm{V})
\end{aligned}
$$

where

$$
\begin{aligned}
& H=L^{2}(0, T ; H) \\
& \left.\left(\tilde{\gamma}_{z}\right)(t)=\gamma^{z(t)} \quad \text { a.e. in }\right]_{0, T}[
\end{aligned}
$$

and $\quad \tilde{\gamma} \in \mathcal{L}\left(\mathrm{L}^{2}(0, \mathrm{~T} ; \mathrm{V}) ; \mathrm{L}^{2}\left(0, \mathrm{~T} ; \mathrm{S}^{\prime}\right)\right)$

$((\cdot, \cdot))$ denotes scalar product between $\mathrm{L}^{2}(0, \mathrm{~T} ; \mathrm{S})$

x/ It is the set of exements $\mathrm{y} \in \mathrm{L}^{2}(0, \mathrm{~T} ; \mathrm{V})$ such that $A(u) y \in L^{2}(0, T ; H)$. 
and $L^{2}(0, T ; s)$.

Let us assume that there exist Hilbert spaces:

$/ 3.8 /$ (i) $\mathrm{w}^{1}(0, \mathrm{~T}) \subset \mathrm{C}(0, \mathrm{~T} ; \mathrm{V})$

injection $W^{1}(0, T) \rightarrow C(O, T ; V)$ is continuous

$/ 3.9 / \quad$ (ii) $\quad \mathrm{Y} \in \mathrm{L}^{2}\left(0, \mathrm{~T} ; \mathrm{S}^{\prime}\right)$

injection $\mathrm{Y} \longrightarrow \mathrm{L}^{2}\left(0, \mathrm{~T} ; \mathrm{S}^{\prime}\right)$ is continuous

such that for any given

$/ 3.10 / \quad y_{0} \in V$

$/ 3.11 /$

f $\in \mathrm{L}^{2}(\mathrm{O}, \mathrm{T} ; \mathrm{H})$

$/ 3.12 /$

$\varphi \in X$

the following state equation has the unique solution $y_{u} \in W^{1}(0, T)$ :

$/ 3.13 /$

$$
\begin{aligned}
& \left(\frac{d y_{u}}{d t}(t), z\right) v^{\prime} v+a_{u}\left(t ; y_{u}(t), z\right) \\
& =(f(t), z)_{H}+\left(\varphi(t), \gamma^{z}\right)_{s^{\prime} s}, \quad \forall z \in V \\
& \text { a.e. in }] 0, T[
\end{aligned}
$$

$/ 3.14 / \quad y_{u}(0)=y_{0}$

\section{$\underline{\text { Remark }}$}

Problem of existence of the spaces $/ \mathbb{B} .8 /, / 3.9 /$ is discussed in [9]. Using /3.7/ we obtain another representation [9] of the system $/ 3.13 /, / 3.14 /$ namely

$$
\frac{d y^{u}}{d t}+A(u) y=1
$$

$/ 3.15 /$

$$
\begin{aligned}
& \sigma(u) y_{u}=\varphi \\
& y_{u}(0)=y_{0}
\end{aligned}
$$

We introduce an optimization problem similar to that in previous 
section. We define cost functional

$/ 3.16 / \quad J(\mathrm{u})=\frac{1}{2}\left\|\mathrm{y}_{\mathrm{u}}(\mathrm{T})-\mathrm{z}_{\mathrm{d}}\right\|_{\mathrm{V}}^{2}+\frac{\varepsilon}{2}\|\mathrm{u}\|_{\mathrm{U}}^{2}, \quad \varepsilon \geqslant 0$

where $z_{d} \in V$ is a given element.

We consider the problem of minimization of the cost functional on a given convex set $U_{a d} \subset U$.

If mappings

$/ 3.17 /$

$U \supset U_{a d} \geqslant u \longmapsto A(u) \in \mathcal{L}\left(W^{1}(0, T) ; L^{2}(0, T ; H)\right)$

$/ 3.18 /$

$\mathrm{U} \supset \mathrm{U}_{\mathrm{ad}} \ni \mathrm{u} \longmapsto \sigma(\mathrm{u}) \in \mathcal{L}\left(\mathrm{W}^{1}(0, \mathrm{~T}) ; \mathrm{Y}\right)$

are locally Lipschitzian and the set $U_{a d} \subset U$ is compact then there exists an optimal control $\hat{u} \in U_{a d}$ such that

$/ 3.19 /$

$$
J(\hat{u}) \leqslant J(u), \quad \forall u \in U_{a d}
$$

Proof is given in [9].

To obtain a simple form of necessary conditions of optimality we introduce [8] the so called generalized adjoint state $(p, r) \in L^{2}(0, x ; H) x Y$ defined at the given point $u \in U_{\text {ad }}$ as a solution of generalized adjoint state equation of the form :

$/ 3.20 /$

$$
\begin{array}{ll}
\left(p, \frac{d w}{d t}+A(u) w\right)_{\mathcal{H}}+(x, \quad \sigma(u) w)_{Y} \\
=-\left(y_{u}(T)-z_{d}, w(T)\right)_{V}, \quad \forall w \in W_{0}^{1}(0, T)
\end{array}
$$

where

$$
w_{0}^{1}(0, w) \Rightarrow\left\{w \in w^{1}(0, n)|w|_{t=0}=0\right\}
$$

It can be shown [8], that there exists the unique solution of $/ 3.20 /$ for each $u \in U_{a d}$.

If $\hat{u} \in U_{\text {ad }}$ is an optimal control, mappings $/ 3.17 /, / 3.18 /$ are 
Frechet differentiable and $(\hat{p}, \hat{r})$ is a generalized adjoint state at $\hat{u} \in U_{\text {ad }}$ then necessary conditions of optimality takes on the form: $/ 3.21 /$

$$
\begin{aligned}
& \left(\left\langle A_{u}(\hat{u}) ; u-u\right\rangle y_{\hat{u}}, \hat{p}\right)_{\mathcal{H}} \\
& +\left(\left\langle\sigma_{u}(\hat{u}) ; u-\hat{u}\right\rangle y_{\hat{u}}, \hat{r}\right)_{Y}+\varepsilon(\hat{u}, u-\hat{u})_{U} \geqslant 0 \quad, \quad \forall u \in u_{a d}
\end{aligned}
$$

where $A_{u}(\hat{u}), \sigma_{u}(\hat{u})$ denotes Frechet derivatives of mappings /3.17/ $/ 3.18 /$ taken at optimal point $\hat{u} \in U_{a d}$.

\section{Example:}

Let $\Omega$ be an open region in $\mathrm{R}^{\mathrm{n}}$ with smooth boundary $\Gamma=\partial \Omega$

We introduce the following functional spaces :

(i) $\quad \mathrm{V}=\mathrm{H}^{1}(\Omega), \mathrm{H}=\mathrm{L}^{2}(\Omega)$

(ii) $\quad W^{1}(0, T)=\mathrm{H}^{2}, 1(\mathrm{Q})$

where

$$
\mathrm{Q}=\Omega \times] 0, \mathrm{~T}[\quad, \quad \mathrm{~T}>0
$$

$$
\text { (iii) } \quad \mathrm{Y}=\mathrm{H}^{1 / 2,1 / 4}(\Sigma)
$$

where

$$
\Sigma=\Gamma \times] 0, T[
$$

(iv)

$$
D=H^{1}(\Omega ; \Delta)
$$

where

$$
\begin{aligned}
& H^{1}(\Omega ; \Delta)=\left\{y \in H^{1}(\Omega) \mid y \in L^{2}(\Omega)\right\} \\
& s=H^{1 / 2}(\Gamma)
\end{aligned}
$$

We define the set of admissible controls $U_{\text {ad }}<U=H^{2}(0, T)$ as the set of solutions of ordinary differential equation

$$
\left\{\begin{array}{l}
\frac{d u}{d t}=-a_{1} u+v_{1} \\
\frac{d v_{1}}{d t}=-a_{2} v_{1}+v \\
u(0)=v_{1}(0)=0
\end{array}\right.
$$

for all $v \in L^{2}(0, T)$ such that 


$$
0 \leqslant v(t) \leqslant 1 \quad \text { a.e. in }] 0, \mathrm{~T}[
$$

where $a_{1}, a_{2}>0$ are given constants.

Let there be given real functions $F(),. g($.$) such that$

$$
\begin{array}{ll}
\text { (i) } & F(.), g(.) \in C^{2}[0,1] \\
\text { (ii) } & F(r) \geqslant \alpha>0, \quad \forall r \in[0,1] \\
& g(r) \geqslant 0 \quad, \quad \forall r \in[0,1]
\end{array}
$$

We introduce the state equation of the form:

$$
\begin{gathered}
\int_{\Omega} \frac{\partial y_{u}}{\partial t} z d \Omega+F(u(t)) \sum_{i=1}^{n} \int_{\Omega} \frac{\partial y_{u}}{\partial x_{i}} \frac{\partial z}{\partial x_{i}} d \Omega \\
+g(u(t)) \int_{\Gamma} y_{u} z d \Gamma=\int_{\Omega} f d \Omega+\int_{\Gamma} \varphi_{z} d \Gamma \\
\forall z \in H^{1}(\Omega), \\
\left.y_{u}(x, 0)=y_{0}(x), \quad \text { in }\right] 0, T[
\end{gathered}
$$

It can be shown that for given

$$
\begin{gathered}
\varphi \in H^{1 / 2,1 / 4}(Z) \\
f \in L^{2}(Q) \\
y_{0} \in H^{1}(\Omega) \\
u \in U_{a d}
\end{gathered}
$$

There exists the unique solution $y_{u} \in \mathrm{H}^{2,1}(\mathrm{Q})$ of the above problem.

In this case operators $/ 3.17 /, / 3.18 /$ have the form

$$
\begin{array}{ll}
A(u) y=F(u) \Delta y, & y \in D=H^{1}(\Omega ; \Delta) \\
\sigma(u) y=\frac{\partial y}{\partial n}+g(u) y &
\end{array}
$$

where $\frac{\partial}{\partial n}$ denotes normal derivative to the boundary $\Gamma=\partial \Omega$ 
We introduce the cos $t$ functional of the form

$$
J(u)=\frac{1}{2}\left\|y_{u}(T)-z_{a}\right\|_{H^{1}(\Omega)}^{2}+\frac{\varepsilon}{2}\|u\|_{H^{2}(O, T)}^{2}
$$

where $\varepsilon \geqslant 0$ and $z_{d} \in H^{1}(\Omega)$ is a given element.

For any given $\varepsilon \geqslant 0$ there exists [9] an optimal control $\hat{u} \in U_{\text {ad }}$ which is characterized by the following inequality :

$$
\begin{aligned}
& \int_{Q}\left[\frac{d F}{d u}(\hat{u})(u-u)\right] \Delta y_{\hat{u}} \hat{p} d Q \\
& +\int_{\Sigma}\left[\frac{d F}{d u}(\hat{u}) \frac{\partial y \hat{u}}{\partial n}+\frac{d g}{d u}(\hat{u}) y_{\hat{u}}\right](u-\hat{u}) \hat{r} d \Sigma \\
& +\varepsilon(\hat{u}, u-\hat{u})_{H^{2}(0, T)} \geqslant 0, \quad \forall u \in U_{a d}
\end{aligned}
$$

where $(\hat{p}, \hat{r}) \in L^{2}(Q) \times\left(H^{1 / 2,1 / 4}(\Sigma)\right)^{\prime}$ is the unique solution $[9]$ of the problem:

$$
\begin{aligned}
& \int_{\hat{Q}}\left(\frac{d w}{d t}+F(\hat{u}) \Delta w\right) \hat{p} d Q \\
& +\int_{\Sigma}\left(F(\hat{u}) \frac{\partial w}{\partial n}+g(\hat{u}) w\right) \hat{r} d \Sigma \\
& =-\left(y_{u}(\cdot, T)-{ }_{d} d, w(\cdot, T)\right)_{H^{1}(\Omega) \quad \forall w \in H^{2}, 1(Q)}
\end{aligned}
$$

such that $(\ldots, 0)=0$

where we use the same notation for the scalar product in $L^{2}(\Sigma)$ and the scalar product between $H^{1 / 2,1 / 4}(\Sigma)$ and $\left(H^{1 / 2,1 / 4}(\Sigma)\right)^{\prime}$. 


\section{References}

[1] Aubin J.P. : Approximation of Elliptic Boundary Value Problems, Wiley, Interscience 1972

[2] Chavent G. : These, Paris 1971.

[3] Lions J.P. : Contrôle optimale de systemes gouvernés par des équations aux derivées partielles, Dunod, Paris 1968.

[4] Lions J.P., Magenes F. : Problèmes aux limites non homogenes et applications, Vol. 1,2, Dunod, Paris 1968.

[5] Fleming W.H. : Optimal control of partially observable ditfussion, SIAM J. Control 6, 1968.

[6] Ladyzhenskaja 0,A. : Krajewyje zadachi matematicheskoj fizyki, Nauka, 1973 /in Russian/.

[7] Sokołowski J. : On parametric optimal control for a class of linear and quasilinear equations of parabolic type, Control and Cyrernetics 1, 1975.

[8] Sokołowski J. : On parametric otpimal control for weak som lutions of abstract parabolio equations, Control and Cybernetics /to appear/.

[9] Sokolowski J. : Thesis, Warsaw 1975.

[10] Tartar L. : Problèmes de contrôle des coefficients dans des équations aux derivées partielles, in Leotures Notes /Control Theory/ Vol. 107, Springer Verlag 1975.

[11] Zollezi T. : Necessary conditions for optimal controls of elliptic or parabolic problems, SIAM J. Control, Vol. 10, No 4,1972 . 